\title{
Ferromagnetic resonance study of thin film antidot arrays: Experiment and micromagnetic simulations
}

\author{
O. N. Martyanov, ${ }^{1}$ V. F. Yudanov, ${ }^{1}$ R. N. Lee, ${ }^{2}$ S. A. Nepijko, $,{ }^{3,4}, *$ H. J. Elmers, ${ }^{4}$ R. Hertel, ${ }^{5}$ C. M. Schneider,${ }^{5}$ and \\ G. Schönhense ${ }^{4}$ \\ ${ }^{1}$ Boreskov Institute of Catalysis, Siberian Branch of the Russian Academy of Sciences, Prospekt Akademika Lavrentieva 5, \\ 630090 Novosibirsk, C.I.S./Russia \\ ${ }^{2}$ Budker Institute of Nuclear Physics, Siberian Branch of the Russian Academy of Sciences, Prospekt Akademika Lavrentieva 11, \\ 630090 Novosibirsk, C.I.S./Russia \\ ${ }^{3}$ Institute of Physics, National Academy of Sciences of Ukraine, Prospekt Nauki 46, 03028 Kiev, C.I.S./Ukraine \\ ${ }^{4}$ Institute of Physics, University Mainz, Staudingerweg 7, 55099 Mainz, Germany \\ ${ }^{5}$ Institute of Solid State Research IFF-6, Electronic Properties, Research Centre Jülich, D-52425 Jülich, Germany
}

(Received 18 July 2006; revised manuscript received 8 February 2007; published 21 May 2007)

\begin{abstract}
The dynamic magnetic properties of two-dimensional periodic Co antidot arrays were studied by $X$-band ferromagnetic resonance. The experimental results on geometrically scaled antidot arrays reveal a strong attenuation of the uniform ferromagnetic resonance mode in comparison to a continuous film, but an excitation of nonuniform in-plane spin-wave modes. Micromagnetic finite-element simulations show that the static magnetic structure in an antidot array depends on the direction of the external field with respect to the symmetry axes of the antidot lattice, even if the external field is strong enough to enforce a technically saturated magnetization state. The analysis gives evidence that characteristic inhomogeneities in the magnetization distribution around the antidots give rise to the changes of the resonance modes with the in-plane direction of the magnetization.
\end{abstract}

DOI: $10.1103 /$ PhysRevB.75.174429

\section{INTRODUCTION}

The dynamic properties of magnetic nanostructures are drawing increasing attention ${ }^{1}$ due to the developments in information technology with respect to storage density and data throughput. The importance of the geometric confinement of the magnetization and its drastic impact on the properties of magnetic nanoparticles ${ }^{2}$ has been the topic of several investigations. Besides the static and quasistatic properties, also the magnetization dynamics in nanopatterned particles has recently attracted considerable interest. ${ }^{3-5}$ In addition to numerous studies of the influence of nanopatterning on the magnetization in mesoscopic magnetic dots, several studies have also addressed the issue for antidot arrays. While the static magnetic properties of antidot arrays ${ }^{6-9}$ have been studied to a good extent, not much is known about their dynamic properties up to now. Such antidot arrays have been proposed for high-density storage media, ${ }^{10}$ because they are expected to provide a higher thermal stability due to the amount of magnetic material than "positive" arrays of magnetic particles of the same dimension as the antidots.

One of the interesting dynamic phenomena is the branching of spin-wave excitations, which arises as a result of quantization in small structures when the feature size becomes comparable to the wavelength of the spin waves. Considerable efforts were made to investigate dynamic properties of scaled arrays of cylindrical magnetic dots and wires by Brillouin light scattering and other techniques. ${ }^{11-13}$ On the other hand, only a few ferromagnetic resonance (FMR) experiments have been performed on nanoscaled magnets ${ }^{14-18}$ up to now, although FMR is a powerful tool for investigating standing spin-wave spectra. ${ }^{18}$ Recently, some authors using FMR (Ref. 19) have revealed unusual properties of antidot Permalloy structures with a rectangular unit cell. They ob-
PACS number(s): 75.70.Ak, 75.75.+a, 76.50.+g, 75.40.Gb

served localized modes in the region between the antidots inferred by spatially averaged ferromagnetic resonance. In the present work, we studied the FMR response of scaled two-dimensional periodic Co circular-shaped antidot arrays possessing square symmetry with varying period length. We found a strong attenuation of the uniform ferromagnetic resonance of such patterned films compared to continuous films accompanied by an excitation of nonuniform standing spin waves.

\section{EXPERIMENTAL SETUP}

We investigated microstructured Co films and a continuous Co film as a reference sample. $\mathrm{Si}(100)$ single-crystal surfaces covered by native oxide served as substrates. A Co film of $d \approx 20 \mathrm{~nm}$ thickness was deposited onto a 7-nm-thick Ta buffer layer and covered by a 3-nm-thick $\mathrm{Cu}$ protective film. This film stack was microstructured by deep ultraviolet photolithography and ion milling. The quality of the resulting two-dimensional periodic Co antidot array (i.e., shape and size of the holes, as well as distances between them) was controlled by a scanning electron microscope (SEM) (Jeol JSM 6300). A typical structure is shown in Fig. 1. As can be seen, the holes are shaped like round disks. The ratio of the hole radius $r$ to the array period length $a$ was chosen as 0.3 . We studied two samples with a period length of $a=1.2$ and $0.8 \mu \mathrm{m}$. All samples (continuous and microstructured ferromagnetic films) were cut into $4 \times 4 \mathrm{~mm}^{2}$ platelets. In the case of the microstructured ferromagnetic films (twodimensional antidot periodic structures), the platelet edges were parallel to the translation vectors with the period length $a$.

The FMR spectra were recorded with a Bruker-EMX $3 \mathrm{~cm}$ spectrometer $(\sim 10 \mathrm{GHz})$. The specimens were placed 


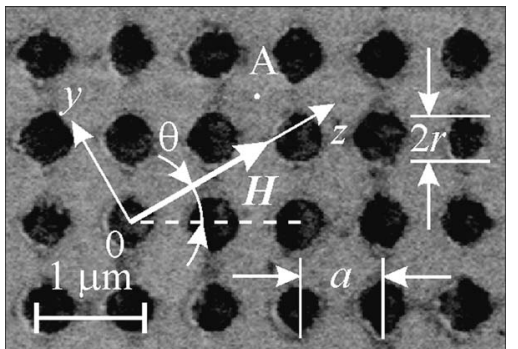

FIG. 1. SEM image of the two-dimensional periodic Co antidot arrays with schematic drawing of the experimental geometry. Here, the $y$ and $z$ axes as well as the external magnetic field $H$ are in the substrate plane, $a$ is the translation vector.

at the center of a rectangular $\mathrm{TE}_{102}$ cavity of the device, the magnetic component of microwave field being perpendicular to the constant external magnetic field. The first derivative of the absorption spectrum was registered at a fixed field modulation frequency of $100 \mathrm{kHz}$. The platelet orientation relative to the external magnetic field was varied with a two-axis goniometer of $\sim 1^{\circ}$ accuracy. We introduce a Cartesian coordinate system with the $z$ axis directed along the external magnetic field $H$ and the $y$ axis directed along the magnetic component of the microwave field. The platelet plane was parallel to the $y z$ plane. During experiments the plate was rotating around the $x$ axis that led to the changes of the angle $\theta$ between external magnetic field direction and translation vector with the period length $a$ (Fig. 1).

\section{EXPERIMENTAL RESULTS}

The FMR spectrum of the continuous ferromagnetic film with the external field applied in the film plane consists of a single line, which corresponds to the uniform resonance mode. The resonant microwave adsorption of the $20 \mathrm{~nm}$ Co film occurs at a magnetic field of $595 \mathrm{G}$ [Fig. 2(a)]. This value results in an effective saturation magnetization of the material of about $1660 \mathrm{G}$ using Kittel's formula. ${ }^{11}$ A rotation

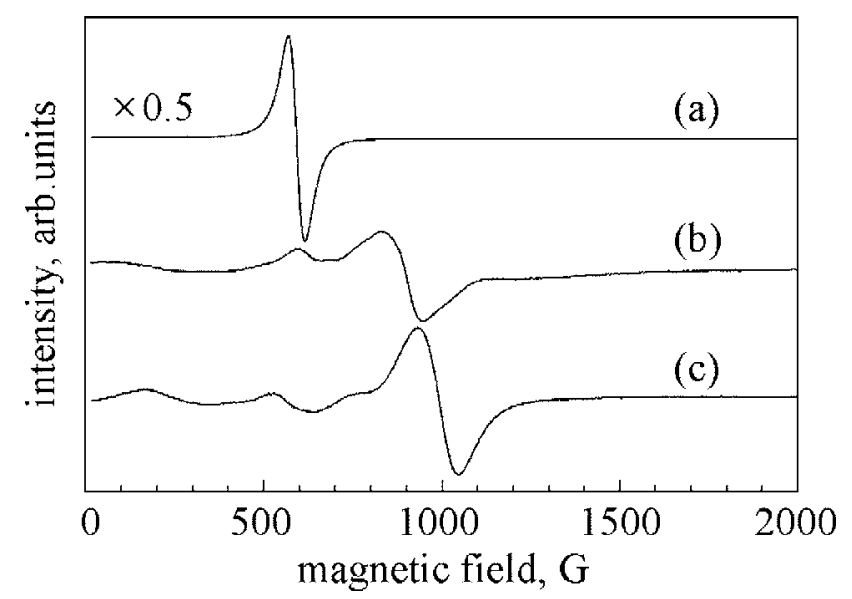

FIG. 2. (a) The FMR spectra in parallel orientation $\left(\theta=0^{\circ}\right)$ of the original continuous film and two-dimensional periodic Co antidot arrays with round-shaped holes with radius $r=0.3 a$ and period length (b) $a=0.8$ and (c) $1.2 \mu \mathrm{m}$.

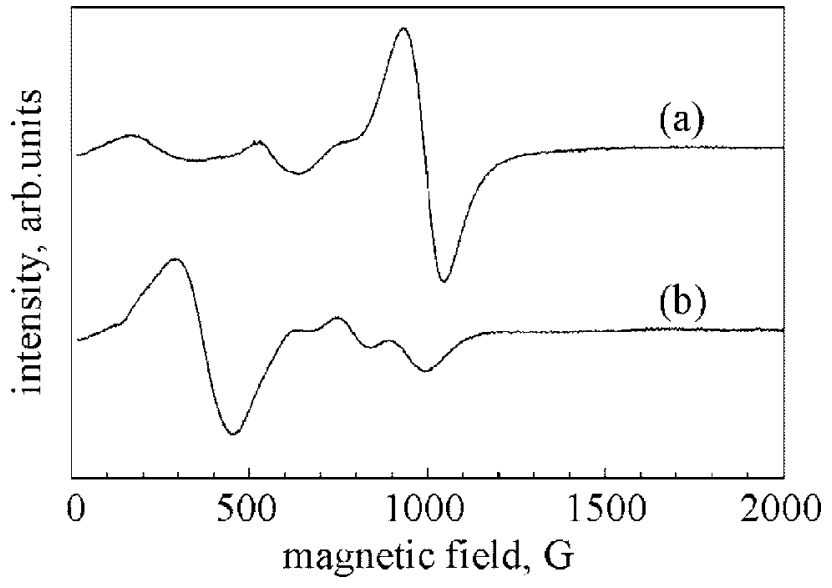

FIG. 3. The FMR spectra of two-dimensional periodic Co antidot arrays with round-shaped holes with $1.2 \mu \mathrm{m}$ period length at different orientation $\theta=0$ (a) and $30^{\circ}$ (b).

of a square extended thin plate in the plane parallel to the external magnetic field should not give rise to any significant changes in the uniform ferromagnetic resonance line, as the demagnetizing factor of the square plate and thus its contribution to the effective anisotropy is small. Experimentally the uniform resonance line position for the continuous Co film changes only within $\sim 40 \mathrm{G}$, primarily depending on the accuracy limit for adjusting the specimen in the spectrometer cavity.

Figures 2(b) and 2(c) show the spectra of the twodimensional periodic Co antidot arrays with two different periodicities at parallel orientation relative to the external magnetic field. In comparison with the continuous film spectrum [Fig. 2(a)], there is no strong symmetric line in the spectra of the parallel-oriented antidot arrays in the region near $H \approx 590 \mathrm{G}$ [Figs. 2(b) and 2(c)] that one could unambiguously relate to the uniform resonance observed for continuous film. In this case, the most intense line is observed at higher magnetic fields of 900 and $1000 \mathrm{G}$ for samples with period lengths $a$ of 0.8 and $1.2 \mu \mathrm{m}$, respectively. We also find multipeak absorptions in a wide range of magnetic fields below $1000 \mathrm{G}$.

A change of the in-plane direction of the external field leads to a drastic change of the ferromagnetic resonance spectra. For a specimen rotation by $\theta=30^{\circ}$ (Fig. 3), the most intensive line for the structure with the period length of $1.2 \mu \mathrm{m}$ is now observed at a lower field of $H \approx 370 \mathrm{G}$. The resonance at $1000 \mathrm{G}$ observed for the field orientation along the edge of the unit cell almost disappeared. No pronounced line appears in the region near $600 \mathrm{G}$ corresponding to the uniform resonance of the continuous film.

The attenuation of the uniform FMR mode is one important question; to answer it, micromagnetic modeling was performed (see Sec. IV). Qualitative theoretical analysis shows that the presence of the periodically located nonmagnetic areas (holes) in parallel oriented antidot structure leads to the appearance of an energy-band structure in the power spectrum. When the film is patterned with an antidot array, both the dc and rf fields become spatially nonuniform, with a periodic spatial variation with $a$. Consequently, the spatial 


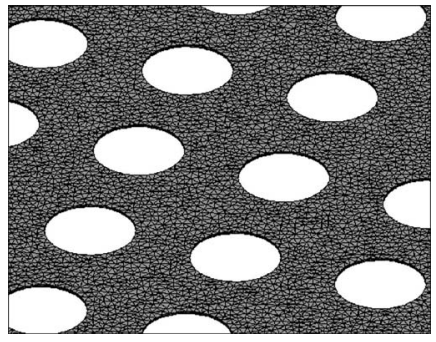

FIG. 4. Perspective view on a mesh of tetrahedral finite elements as used in the simulations to model the static magnetization structure in an array of antidots.

Fourier transforms of these fields have peaks at a wave number $k=\pi / a$, which is in our case on the order of $3 \times 10^{4} \mathrm{~cm}^{-1}$. It is known that the law of dispersion for spin waves in the two-dimensional system at the small value of wave vector $k$ is linear with respect to $k$, and the resonance frequency within the limits of one band can substantially change. With such a low $k$, the dipolar energy dominates over the exchange interaction that results in observation of absorption in the wide range of magnetic fields.

For the Co structures under study, this value is comparable with the value of the field corresponding to the resonance field of continuous plate. Thus, one can observe some kind of effective broadening of resonance absorption line. This broadening is a result of relaxation processes that are induced by the spatial structure. In this case, the broadening mechanism is a two-magnon process, which scatters energy from the uniform mode to other degenerate modes with higher wave numbers. ${ }^{20,21}$ At the same time, the rotation of the antidot structure in its own plane can cause a change of the energy-band structure strong enough to result in dramatic change of the observed spectrum of the resonance absorption. This is due to the fact that when changing the external magnetic field, spin waves belonging to a certain energy band can meet the condition of resonance or get out of it.

From comparison of resonance absorption for the first and the subsequent scans, we can conclude that our sample is close to saturation already at magnetic field strength of $\sim 150 \mathrm{G}$ (at parallel orientation). Nevertheless, even at $\sim 500-1000 \mathrm{G}$ the sample is still not completely homogeneous; that complicates the observed picture.

\section{MICROMAGNETIC MODELING}

Micromagnetic simulations have been employed to study the static magnetic structure of the Co antidot arrays. The simulations have been performed with a finite-element algorithm described in more detail in Ref. 22. Owing to the finite-element discretization, where the magnetic part is subdivided into an irregular mesh of tetrahedral elements, the circular shape of the antidots can be approximated very smoothly (cf. Fig. 4). In order to unambiguously detect anisotropies that result from the array and its influence on the magnetic structure, this feature is particularly important. Other numerical methods, such as the ordinary finitedifference scheme, usually require a "staircase" approximation $^{23}$ in the case of curved surfaces, as they occur (a)
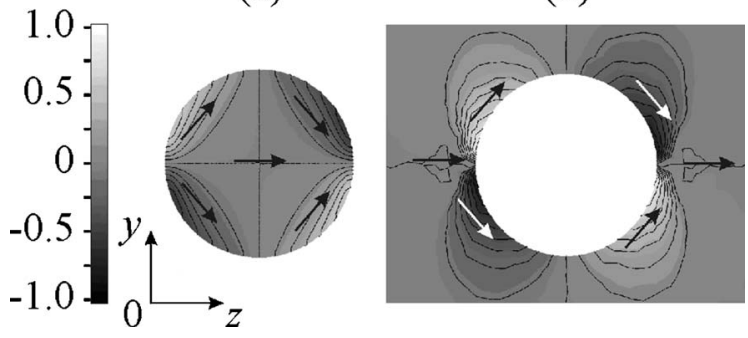

FIG. 5. (a) Onion state in a disk-shaped Co disc with $r=360 \mathrm{~nm}$. (b) "Antionion" or "butterfly" state of the magnetization in the vicinity of a circular antidot of the same size in a Co film. In both cases, the film thickness is $20 \mathrm{~nm}$. An in-plane field of $1000 \mathrm{G}$ is applied along the $z$ axis. The gray scale and the contour lines represent the $y$ component of the magnetization $m_{y}$. The local orientation of the magnetization is schematically represented by the arrows.

here in the circular antidots. The staircase approximation has been reported to give rise to artificial anisotropy effects ${ }^{12,23}$ depending on the choice of the direction of the grid of discretization cells. In the simulations, the dipolar field is calculated with a hybrid scheme that combines the finite element method and the boundary element method. ${ }^{24}$

The simulated sample is a 20 -nm-thick Co film with circular antidots placed on a square lattice. The antidot radius is $r=360 \mathrm{~nm}$ and the period length is $a=1.2 \mu \mathrm{m}$. The material parameters used in the simulation correspond to the regular values for bulk polycrystalline Co metal. ${ }^{25}$ An array of $5 \times 5$ antidots has been used for the simulation. It was embedded into a disk of $12 \mu \mathrm{m}$ diameter. A finite-element mesh with variable cell size has been used to reliably resolve the magnetization dynamics in the vicinity of the antidots.

The equilibrium distribution of the magnetization is calculated by means of energy minimization. An external magnetic field of $1000 \mathrm{G}$ is applied in the film plane. The order of magnitude of this external field corresponds to the field used in the experimental FMR study. The field is strong enough to technically saturate the sample. Therefore, the magnetization in the sample is in a unique, well-defined state. This is in contrast to the zero-field case, where a large variety of metastable magnetization configurations can occur. 8,9

\section{A. Static magnetic structure}

Let us first consider the magnetic structure in the vicinity of a single antidot. The magnetization in the film is mostly aligned parallel to the external field. Only in a small region close to the antidot, characteristic inhomogeneities in the magnetization occur. Since the magnetization tends to avoid magnetic surface charges, ${ }^{26}$ it partially aligns with the boundary of the antidot. The magnetic structure around the antidot could be described as an "antionion" state (cf. Fig. 5). The onion state [Fig. 5(a)] is an almost homogeneous state that has been found in magnetic rings ${ }^{27}$ and disks. ${ }^{28}$ There are four inhomogeneous regions around each antidot, as can be clearly seen from the contour lines of constant values of 


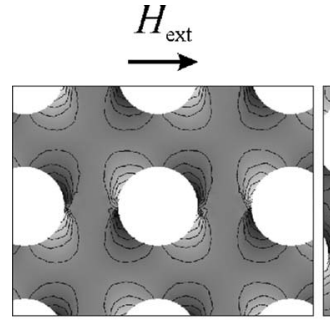

(a)

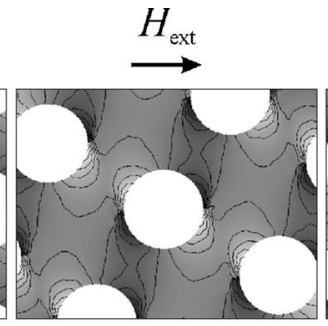

(b)

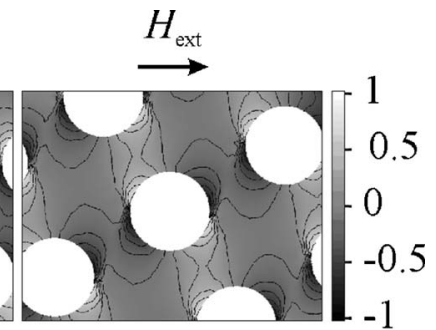

(c)

FIG. 6. Simulated magnetic structure in an antidot array as described in the text. (a) The antidot array is oriented parallel to the external field. The butterfly structures around the antidots are decoupled from the inhomogeneities around neighboring antidots. (b) The antidot array is rotated by $\theta=30^{\circ}$. The inhomogeneities of the antionions are now connected. (c) The micromagnetic structure changes accordingly when the array is rotated by $\theta=-30^{\circ}$. In all cases, the film thickness is $20 \mathrm{~nm}$ and a field of $1000 \mathrm{G}$ is applied parallel to the $z$ axis.

the $y$ component of the magnetization $m_{y}$ in Fig. 5(b). In spite of the analogy to the onion state, the term antionion state for the magnetic structure around the antidot appears to be too clumsy. We therefore prefer to label this a butterfly state, since the shape of the inhomogeneous regions and their fourfold symmetry are reminiscent of the wings of a butterfly.

In a single, isolated antidot in a homogeneous and isotropic magnetic film, the axis of the butterfly state is obviously parallel to the direction of the external field. In the case of a regular array of antidots, however, the fourfold anisotropy of the butterfly state interacts with the anisotropy due to the lattice of antidots. This is illustrated in three examples of a square array of antidots shown in Fig. 6. In all cases, the field is applied in the $z$ direction. If this direction coincides with an axis of the antidot lattice [Fig. 6(a)], there is hardly any cross-talk between the inhomogeneities of neighboring antidots. In this case, the antidots can be considered as rather isolated entities, at least as far as the static magnetic structure is concerned.

This situation changes when the antidot array (or the external field direction) is rotated by $30^{\circ}$ in the film plane [cf. Fig. 6(b)]. While the axis of the butterfly state around each antidot essentially remains aligned with the external field direction, the overall magnetic structure is significantly different. By rotating the antidot lattice with respect to the external field, the distance between the inhomogeneities around neighboring antidots is changed. Staying in the picture of a butterfly structure, one could say that the wings of neighboring butterflies are now touching each other. This connection of the formerly isolated inhomogeneities leads to a modulation of the overall magnetic structure by the occurrence of stripes between the antidots, as can be seen in the dark regions in Fig. 6(b). This type of magnetic domain structure is typical for magnetic circular antidot arrays. ${ }^{29-31}$ The qualitatively same effect occurs if the lattice is rotated by $30^{\circ}$ in the opposite direction, where the dark stripes are replaced by bright stripes [cf. Fig. 6(c)].

These examples demonstrate that the static magnetic structure in an antidot array can depend on the direction of the external field with respect to the antidot lattice, even if the external field is strong enough to enforce a technically saturated magnetization state. The static micromagnetic modeling already indicates that the dynamic response of the magnetization in a FMR experiment could be anisotropic with respect to the external field direction.

\section{B. Magnetization dynamics}

To investigate the dynamic response of the magnetization in the antidot array, we have performed micromagnetic simulations based on the Landau-Lifshitz-Gilbert equation. A static external in-plane field of $1000 \mathrm{G}$ was applied parallel to the $z$ direction, i.e., one of the axes of the antidot array. The magnetization dynamics after a short perturbation has been simulated by applying a field pulse of Gaussian shape (peak: $100 \mathrm{G}, 10$ ps width) along the $y$ direction. The Gilbert damping constant was set to $\alpha=0.01$ after the perturbation, the magnetic system starts to oscillate incoherently. Different, well-defined normal modes of the magnetization dynamics have been found by means of a Fourier analysis. ${ }^{32}$

A pronounced peak in the spectrum of the magnetic oscillations is observed at $10.5 \mathrm{GHz}$. The Fourier analysis of the magnetization dynamics at this frequency shows that this peak is related to periodic oscillations of the $z$ component of the magnetization $m_{z}$ in the regions previously labeled as the "butterfly wings," cf. Fig. 7(a), while the $y$ component of the magnetization $m_{y}$ oscillates in striped regions with a width of approximately the radius of the antidots. These stripes are oriented along the static field direction, and they are located between the head and the tail of neighboring butterfly structures [cf. Fig. 7(b)]. The oscillation amplitude of $m_{y}$ is in this mode much higher than the oscillation amplitude of $m_{z}$. We found a further resonant mode at $21 \mathrm{GHz}$. At this frequency, the oscillation pattern of $m_{z}$ [Fig. $\left.7(\mathrm{c})\right]$ is very similar to the previously described oscillation pattern of $m_{y}$ in the $10.5 \mathrm{GHz}$ mode, and vice versa [see Fig. 7(d)]. Contrary to the $10.5 \mathrm{GHz}$ mode, where the oscillation is mostly pronounced in the $m_{y}$ component, both the $m_{y}$ and the $m_{z}$ components now oscillate with similar amplitude.

We have also simulated the magnetization dynamics with an external field value of $1000 \mathrm{G}$, after tilting the antidot array by $30^{\circ}$ with respect to the external field direction. In this case, the main resonant mode is found at $11.5 \mathrm{GHz}$, while the next-higher mode is at $23 \mathrm{GHz}$. Also in this case, the pattern of the oscillations in $m_{y}$ of the lower-frequency mode is similar to the $m_{z}$ oscillation pattern of the higherfrequency mode. 


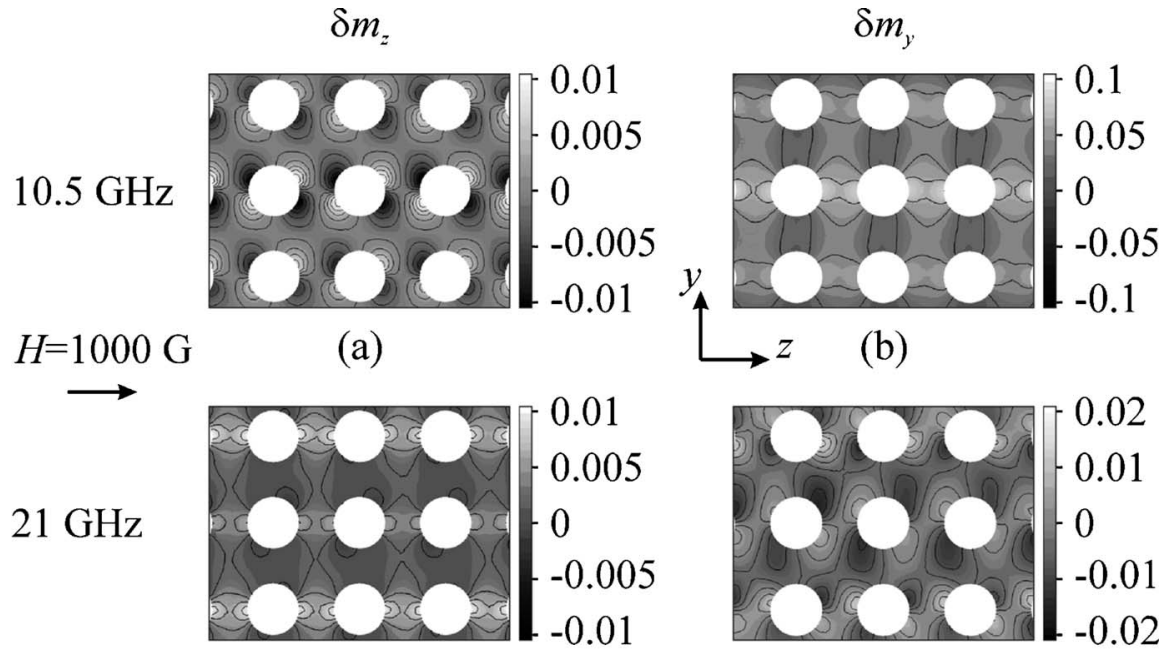

(c)

(d)

FIG. 7. Resonant modes of the antidot array in the case of $H=1000 \mathrm{G}$ applied parallel to the array axis. The main oscillation mode $m_{z}$ and $m_{y}$ at $10.5 \mathrm{GHz}$ is displayed in the panels (a) and (b), respectively. Panels (c) and (d) describe the second resonant mode $m_{z}$ and $m_{y}$, occurring at a resonance frequency of $21 \mathrm{GHz}$. These images were obtained by means of a Fourier transform of the simulated magnetization dynamics, followed by a windowed Fourier back transformation from frequency space into the time domain. The frequencies are selected by applying a small window around the frequency of interest prior to the back transformation. The gray scale and the contour lines represent the region of largest oscillation amplitudes.

Simulations for an external in-plane field of $H=350 \mathrm{G}$ and orientation of the antidot array $\theta=0^{\circ}$ and $30^{\circ}$ reveal a different behavior.

Figure 8 shows the results for the Fourier transformation of $m_{y}$ in point A (see Fig. 1) for (a) $H=1000$ and (b) $350 \mathrm{G}$. Here, the solid and dotted lines correspond to $\theta=0^{\circ}$ and $30^{\circ}$, respectively. As can be seen from Figs. 8(a) and 8(b), the frequencies of resonance modes depend on the value of the static external in-plane field $H$ and of the angle $\theta$. The resonance modes as indicated in Figs. 8(a) and 8(b) are 10.5 and $21 \mathrm{GHz}\left(H=1000 \mathrm{G}, \theta=0^{\circ}\right) ; 11.5$ and $23 \mathrm{GHz}(H=1000 \mathrm{G}$, $\left.\theta=30^{\circ}\right) ; 5.5,11$, and $16.5 \mathrm{GHz}\left(H=350 \mathrm{G}, \theta=0^{\circ}\right)$; and 6.5 , 13 , and $19.5 \mathrm{GHz}\left(H=350 \mathrm{G}, \theta=30^{\circ}\right)$ with an accuracy of $\pm 1 \mathrm{GHz}$. Note that absolute values strongly depend on the thickness. Deviations of the actual sample thickness from the nominal value of $20 \mathrm{~nm}$ make a direct comparison to experimental values difficult. Further problems occurring for the micromagnetic simulation of FMR experiments are reported, as given in Ref. 33 .

The amplitude of the Fourier spectrum corresponds to the degree of microwave absorption in the FMR experiment. Apart from a quantitative comparison, the simulation agrees well with the experimental data. A main resonance frequency shows up for an external field of $1000 \mathrm{G}$ at $\sim 10 \mathrm{GHz}$ [Fig. $8(\mathrm{a})]$ in accordance with the resonant absorption in the FMR experiment. This absorption is observed for $\theta=0^{\circ}$ and $30^{\circ}$ (Fig. 3). For an external magnetic field of $350 \mathrm{G}$, a considerable Fourier amplitude at $\sim 10 \mathrm{GHz}$ is observed for the case of $\theta=30^{\circ}$ (although the maximum is at a lower frequency of $\sim 5 \mathrm{GHz}$ ). The amplitude is much less prominent at $\sim 10 \mathrm{GHz}$ for $\theta=0^{\circ}$ [Fig. 8(b)]. This corresponds to the absence of an absorption in the experiment for this case.

Point A was chosen as a representative point, located in the middle between four antidots. Besides this point, oscilla-

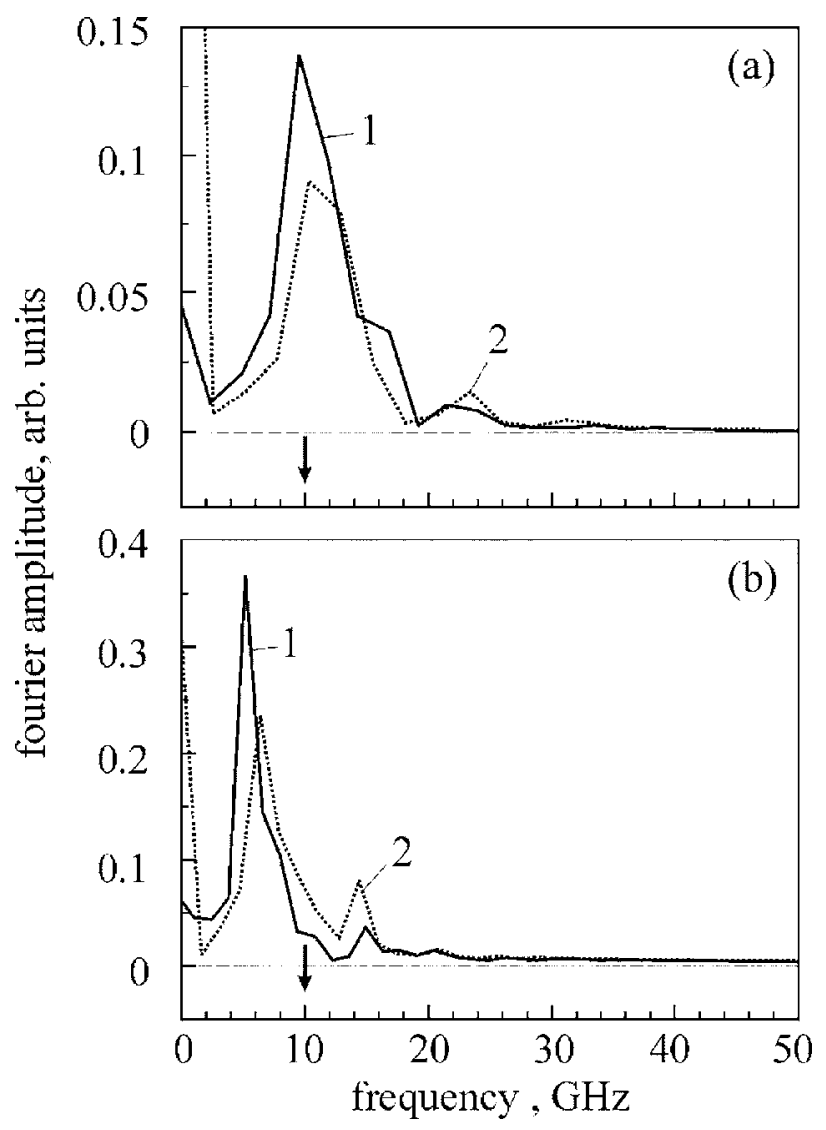

FIG. 8. Fourier transformation of the magnetization component $m_{y}$ in the Co antidot arrays in point A, designated in Fig. 1, for (a) $H=1000$ and (b) $350 \mathrm{G}$. The cases of $\theta=0^{\circ}$ and $30^{\circ}$ are represented by (1) solid and (2) dotted lines, respectively. The arrow denotes the experimental frequency. 
tions have been recorded at two further points. These two additional points are located on very different positions (close to the border of an antidot and in the middle between two antidots). The results show that also at these points the same frequencies are found previously at point $\mathrm{A}$. The most important difference between the Fourier spectra obtained at these points is the Fourier amplitude, while the main frequencies are the same. Thus, the above presented results of Fourier spectra analysis obtained at point A are more general.

In all cases, the simulations show that the frequencies and the oscillation patterns of resonant modes in antidot arrays are strongly affected by the orientation and the absolute value of the external field with respect to the antidot array. The strongly oscillating regions of the magnetic normal modes are mostly located in narrow strips connecting nearest-neighbor antidots. The magnetic microstructure in the immediate vicinity of the antidots, i.e., the butterfly regions, also displays characteristic oscillations, particularly when the external field is aligned parallel to the array axis.

\section{CONCLUSION}

In summary, FMR has been successfully employed to investigate collective spin-wave excitation phenomena in twodimensional periodic Co antidot arrays with different periods. We found a strong unexpected attenuation of the uniform ferromagnetic resonance mode in the antidot array compared to the resonance mode of a continuous film going along with an additional multipeak absorption in a wide range of magnetic fields. The FMR spectra strongly depend on the orientation of the external field relative to the antidot array. A numerical simulation qualitatively agrees with our experimental findings and reveals that the static magnetization structure in an antidot array depends on the direction of the external field with respect to the symmetry axes of the antidot lattice. The strong angular dependence of the FMR spectra indicates the excitation of heterogeneous spin-wave modes in the two-dimensional antidot array.
*FAX: +49-6131-3925412; Email address: nepijko@uni-mainz.de

${ }^{1}$ M. Farle, Rep. Prog. Phys. 61, 755 (1998).

${ }^{2}$ R. P. Cowburn, D. K. Koltsov, A. O. Adeyeye, M. E. Welland, and D. M. Tricker, Phys. Rev. Lett. 83, 1042 (1999).

${ }^{3}$ M. Buess, R. Höllinger, T. Haug, K. Perzlmaier, U. Krey, D. Pescia, M. R. Scheinfein, D. Weiss, and C. H. Back, Phys. Rev. Lett. 93, 077207 (2004).

${ }^{4}$ G. Gubbiotti, G. Carlotti, T. Okuno, M. Grimsditch, L. Giovannini, F. Montoncello, and F. Nizzoli, Phys. Rev. B 72, 184419 (2005).

${ }^{5}$ A. Krasyuk, F. Wegelin, S. A. Nepijko, H. J. Elmers, G. Schönhense, M. Bolte, and C. M. Schneider, Phys. Rev. Lett. 95, 207201 (2005).

${ }^{6}$ L. J. Heyderman, H. H. Solak, F. Nolting, and C. Quitmann, J. Appl. Phys. 95, 6651 (2004).

${ }^{7}$ A. Yu Toporov, R. M. Langford, and A. K. Petford-Long, Appl. Phys. Lett. 77, 3063 (2000).

${ }^{8}$ L. López-Díaz, L. Torres, and J. I. Iñiguez, J. Magn. Magn. Mater. 196-197, 805 (1999).

${ }^{9}$ L. Torres, L. López-Díaz, and J. Iñiguez, Appl. Phys. Lett. 73, 3766 (1998).

${ }^{10}$ R. P. Cowburn, A. O. Adayeye, and J. A. C. Bland, J. Magn. Magn. Mater. 173, 193 (1997).

${ }^{11}$ J. Jorzick, C. Krämer, S. O. Demokritov, B. Hillebrands, B. Bartenlian, C. Chappert, D. Decanini, F. Rousseaux, E. Cambril, E. Søndergard, M. Bailleul, C. Fermon, and A. N. Slavin, J. Appl. Phys. 89, 7091 (2001).

${ }^{12}$ G. Gubbiotti, G. Carlotti, R. Zivieri, F. Nizzoli, T. Okuno, and T. Shinjo, J. Appl. Phys. 93, 7607 (2003)

${ }^{13}$ H. Fritzsche, K. Temst, and M. J. Van Bael, Appl. Phys. A: Mater. Sci. Process. 74, S1535 (2002).

${ }^{14}$ K. Furukawa, D. Shiomi, K. Sato, T. Takui, K. Itoh, A. Maeda, M. Kume, K. Shibata, and I. Nakatani, Mol. Cryst. Liq. Cryst. Sci. Technol., Sect. A 335, 163 (1999).

${ }^{15}$ S. Jung, B. Watkins, L. DeLong, J. B. Ketterson, and V. Chandrasekhar, Phys. Rev. B 66, 132401 (2002).

${ }^{16}$ W. Xu, D. B. Watkins, L. E. DeLong, K. Rivkin, J. B. Ketterson, and V. V. Metlushko, J. Appl. Phys. 95, 6645 (2004).
${ }^{17}$ Ch. Yu, M. J. Pechan, and G. J. Mankey, Appl. Phys. Lett. 83, 3948 (2003).

${ }^{18}$ Ch. Yu, M. J. Pechan, W. A. Burgei, and G. J. Mankey, J. Appl. Phys. 95, 6648 (2004).

${ }^{19}$ M. J. Pechana, Ch. Yu, R. L. Compton, J. P. Park, and P. A. Crowell, J. Appl. Phys. 97, 10J903 (2005).

${ }^{20}$ K. Lenz, H. Wende, W. Kuch, K. Baberschke, K. Nagy, and A. Janossy, Phys. Rev. B 73, 144424 (2006).

${ }^{21}$ D. L. Mills and S. M. Rezende, in Spin Dynamics in Confined Magnetic Structures II, Topics in Applied Physics, Vol. 87, edited by B. Hillebrands and K. Oundjela (Springer, Berlin, 2003), pp. 27-59.

${ }^{22}$ R. Hertel, J. Appl. Phys. 90, 5752 (2001).

${ }^{23}$ C. J. García-Cervera, Z. Gimbutas, and E. Weinan, J. Comput. Phys. 184, 37 (2003).

${ }^{24}$ D. R. Fredkin, T. R. Koehler, J. F. Smyth, and S. Schultz, J. Appl. Phys. 69, 5276 (1991).

${ }^{25}$ M. Kläui, C. A. F. Vaz, J. A. C. Bland, T. L. Monchesky, J. Unguris, E. Bauer, S. Cherifi, S. Heun, A. Locatelli, L. J. Heyderman, and Z. Cui, Phys. Rev. B 68, 134426 (2003).

${ }^{26}$ W. F. Brown, Jr., Micromagnetics (Wiley, New York, 1963).

${ }^{27}$ J. Rothman, M. Kläui, L. López-Díaz, C. A. F. Vaz, A. Bleloch, J. A. C. Bland, Z. Cui, and R. Speaks, Phys. Rev. Lett. 86, 1098 (2001).

${ }^{28}$ J. K. Ha, R. Hertel, and J. Kirschner, Phys. Rev. B 67, 224432 (2003).

${ }^{29}$ C. T. Yu, H. Jiang, L. Shen, P. J. Flanders, and G. J. Mankey, J. Appl. Phys. 87, 6322 (2000).

${ }^{30}$ P. Vavassori, G. Gubbiotti, G. Zangari, C. T. Yu, H. Yin, H. Jiang, and G. J. Mankey, J. Appl. Phys. 91, 7992 (2002).

${ }^{31}$ I. Ruiz-Feal, L. López-Díaz, A. Hirohata, J. Rothman, C. M. Guertler, J. A. C. Bland, L. M. Garcia, J. M. Torres, F. Bartolome, M. Natali, D. Decanini, and Y. Chen, J. Magn. Magn. Mater. 242-245, 597 (2002).

${ }^{32}$ R. Hertel and H. Kronmüller, IEEE Trans. Magn. 34, 3922 (1998).

${ }^{33}$ R. Hertel and J. Kirschner, J. Magn. Magn. Mater. 272-276, 655 (2004). 